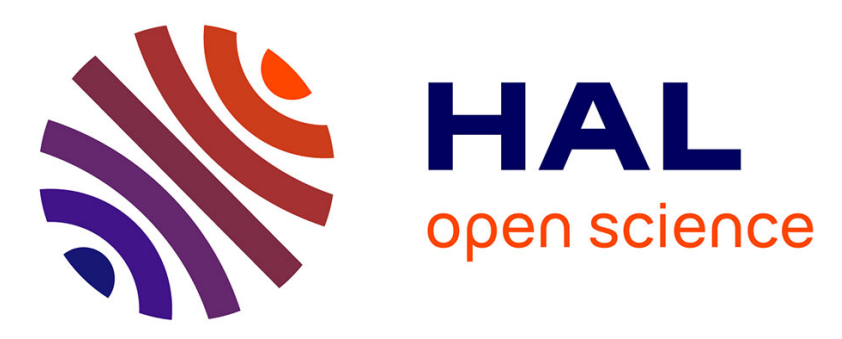

\title{
Exact Outage Probability of a Hybrid Satellite Terrestrial Cooperative System with Best Relay Selection
}

Sokchenda Sreng, Benoît Escrig, Marie-Laure Boucheret

\section{- To cite this version:}

Sokchenda Sreng, Benoît Escrig, Marie-Laure Boucheret. Exact Outage Probability of a Hybrid Satellite Terrestrial Cooperative System with Best Relay Selection. IEEE International Conference on Communications - ICC 2013, Jun 2013, Budapest, Hungary. pp. 4520-4524. hal-01150349

\author{
HAL Id: hal-01150349 \\ https://hal.science/hal-01150349
}

Submitted on 11 May 2015

HAL is a multi-disciplinary open access archive for the deposit and dissemination of scientific research documents, whether they are published or not. The documents may come from teaching and research institutions in France or abroad, or from public or private research centers.
L'archive ouverte pluridisciplinaire HAL, est destinée au dépôt et à la diffusion de documents scientifiques de niveau recherche, publiés ou non, émanant des établissements d'enseignement et de recherche français ou étrangers, des laboratoires publics ou privés. 


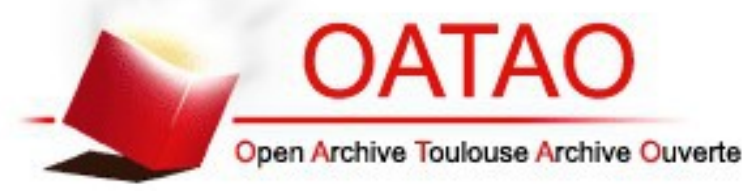

\section{Open Archive TOULOUSE Archive Ouverte (OATAO)}

OATAO is an open access repository that collects the work of Toulouse researchers and makes it freely available over the web where possible.

This is an author-deposited version published in : http://oatao.univ-toulouse.fr/ Eprints ID : 12921

To link to this article : DOI :10.1109/ICC.2013.6655280

URL : http://dx.doi.org/10.1109/ICC.2013.6655280

To cite this version : Sreng, Sokchenda and Escrig, Benoît and Boucheret, Marie-Laure Exact Outage Probability of a Hybrid Satellite Terrestrial Cooperative System with Best Relay Selection. (2013) In: IEEE International Conference on Communications - ICC 2013, 9 June 2013 - 13 June 2013 (Budapest, Hungary).

Any correspondance concerning this service should be sent to the repository administrator: staff-oatao@,listes-diff.inp-toulouse.fr 


\title{
Exact Outage Probability of a Hybrid Satellite Terrestrial Cooperative System with Best Relay Selection
}

\author{
Sokchenda Sreng, Benoît Escrig and Marie-Laure Boucheret \\ University of Toulouse, INPT-ENSEEIHT/IRIT \\ 2 rue Charles Camichel, BP 7122 \\ 31071 Toulouse cedex 7, France \\ Email: \{sokchenda.sreng, benoit.escrig, marie-laure.boucheret\}@enseeiht.fr
}

\begin{abstract}
In this paper, we derive the exact outage probability of a hybrid satellite-terrestrial cooperative system (HSTCS). A selective decode-and-forward scheme is implemented between a source node (the satellite) and a destination node (a terrestrial station), and a selection of the best relay terminal is performed. In this proposed system, a two time-slot scenario is considered. During the first time slot, the satellite is broadcasting the information to the terrestrial relays and the destination. In the second time slot, only the best relay is transmitting toward the destination node. Then, both signals are combined using the maximum ratio combining (MRC) technique. The analytical expression of the outage probability is evaluated and is then verified with the simulation. The results show that our analytical expression matched well to the simulation results.
\end{abstract}

\section{INTRODUCTION}

Satellite systems are used to provide services over a wide coverage area. Their potential applications are in the field of broadcasting, navigation, rescue and disaster relief. However, the coverage area is limited by the masking effect caused by obstacles that block the line of sight (LOS) link between the satellite and a terrestrial user. The masking effect becomes more severe in case of low satellite elevation angles or when the user is indoor. To address this issue, the hybrid satelliteterrestrial cooperative systems (HSTCSs) have been proposed [1].

In an HSTCS, the mobile user can exploit the advantages of spatial diversity by receiving signals from both satellite and terrestrial components. Fixed or mobile gap-fillers are used to relay the satellite signal by implementing amplify-and-forward (AF) or decode-and-forward (DF) transmission schemes. Most of satellite broadcasting systems have been implemented using fixed gap-fillers. However, mobile gap-fillers are needed in emergency cases when the fixed infrastructure is not available. In emergency scenarios (e.g., fire, earthquake, flood and explosion), the existing terrestrial infrastructure has been destroyed. So, an HSTCS is appropriate for updating the information. This allows the rescuers to operate efficiently and in a safe way [2]. In particular, a fast and flexible implementation is needed and this could be provided by deploying mobile gap fillers (vehicle or mobile handheld).

Recently, several cooperative scenarios for HSTCSs have been proposed. In [3], the delay diversity technique for a hybrid satellite-terrestrial DVB-SH system has been studied. In this scenario, the user stations receive different versions of the same signal with different delays: one signal from the satellite and other signals from terrestrial relays. No combiner has been implemented. In [4], space-time codes and rate compatible turbo codes have been implemented to achieve diversity gains and additional coding gains. Recently, another HSTCS has been proposed in [5]. The satellite broadcasts the information to terrestrial users in the first time slot and in the second time slot, non-masked terminals are used to relay the information toward masked terminals.

Although the cooperative diversity techniques can increase the system availability, the additional bandwidth is needed for the relay transmission. To minimize the bandwidth consumption while keeping the maximum diversity order, Bletsas et al. [6] have proposed an opportunistic cooperative protocol in which only one relay is used for forwarding the message to the destination. Two criteria for selecting the best relay have been studied. The first criterion is to choose the relay which maximizes the minimum of the source-relay and the relaydestination channel gain, while the second criterion is based on the maximization of the harmonic mean of both channel gains. It has been shown in [7] that the outage performance of both selection techniques are the same in the high-SNR regime. The bandwidth consumption can be further decreased by implementing on-demand cooperation together with the best relay selection [8].

The outage probability is an important performance measure of communication systems operating over fading channels [9] since it provides valuable insight into the design of communication systems. However, most of the previous articles on HSTCSs have provided only the performance based on the simulations [3], [4] and based on the numerical calculations [10] for the case of AF transmission schemes. The exact closed-form outage probability of HSTCSs has not been derived yet.

In this paper, we investigate the performance in terms of outage probability of an HSTCS with best relay selection. The relay is operating in selective DF mode. The closed- 


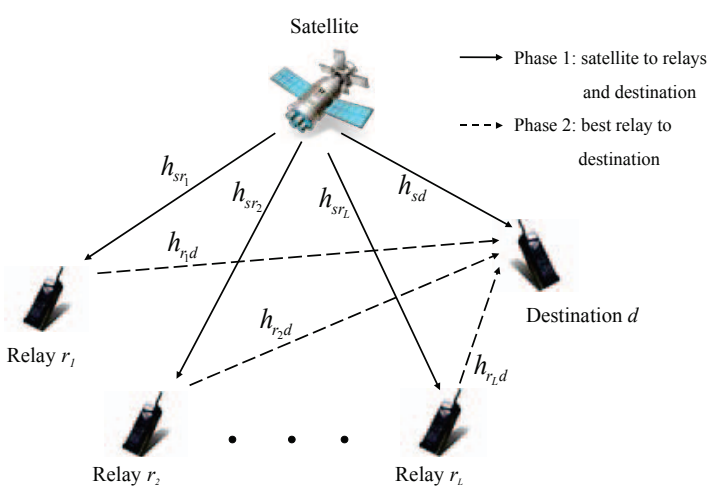

Fig. 1. Hybrid satellite-terrestrial system with $L$ relays and one destination.

form expression of the outage probability is then evaluated using the land mobile satellite (LMS) channel derived in [11]. The analytical results are confirmed using Monte Carlo simulations. The rest of this paper is organized as follows. The system model is discussed in Section II. In Section III, we derived the outage probability of the HSTCS with best relay selection. The simulation and numerical results are presented in Section IV. The conclusion is finally drawn in Section V.

\section{System AND Channel Models}

The system model is represented in Fig. 1. The system consists of one satellite source denoted by $s, L$ terrestrial relays denoted by $r_{1}, r_{2}, \ldots, r_{L}$, and a destination denoted by $d$. We assume that each terminal is equipped with a single antenna and is able to implement the cooperative functionality. The transmission is divided into two phases. In the first phase (broadcasting phase), the satellite broadcasts its signal to the set of $L$ relay nodes and the destination node. We define the decoding set $C$, with cardinality $|C| \leq L$, as the set of relays that can decode the satellite message correctly, i.e., the relay node is said to belong to the decoding set provided that the channel between the source and the relay node is sufficiently good to allow for successful decoding. In the second phase, only one relay is selected from the decoding set $C$ for forwarding the information. Actually, the relay selection takes place between the two phases. The selection can be achieved using the same approach as in [6]. The selected relay is the one that provides the best link quality between a relay and the destination. Then, both signals from the two phases are combined using the MRC technique.

We denote the channel gain between the satellite and the destination by $h_{s d}$, the channel gain between the satellite and the $i^{t h}$ relay by $h_{s r_{i}}$, and the channel gain between the $i^{t h}$ relay and the destination by $h_{r_{i} d}$. We assume that the channels are frequency-flat, independent, but not necessarily identically distributed, fading channels. We also assume that the channel state information (CSI) is perfectly known at the receiver and not known at the transmitter. Furthermore, in our analysis, we assume that the additive white Gaussian noise (AWGN) terms of all links have zero mean and equal variance $N_{0}$. The statistics of the channel models are defined as follows.

- The satellite-destination and the satellite-relays link are modeled as LMS fading channels [11]. The probability density function (PDF), $f_{\left|h_{s x}\right|^{2}}(y)$ of the power channel gain, $\left|h_{s x}\right|^{2}$, is given in [11] for $y \geq 0$ as

$$
\begin{aligned}
f_{\left|h_{s x}\right|^{2}}(y) & =\frac{1}{2 b_{s x}}\left(\frac{2 b_{s x} m_{s x}}{2 b_{s x} m_{s x}+\Omega_{s x}}\right)^{m_{s x}} \exp \left(-\frac{y}{2 b_{s x}}\right) \\
& \times{ }_{1} F_{1}\left(m_{s x} ; 1 ; \frac{\Omega_{s x} y}{2 b_{s x}\left(2 b_{s x} m_{s x}+\Omega_{s x}\right)}\right),
\end{aligned}
$$

where the second subscript $x \equiv d$ and $x \equiv r_{i}$ when we deal with the satellite to the destination and the satellite to the relay $r_{i}$ channels respectively. The parameter $\Omega_{s x}$ is the average power of the LOS component, $2 b_{s x}$ is the average power of the multipath component, and $m_{s x}$ is the Nakagami parameter ranging from 0 to $\infty$. When $m_{s x}=0$, the PDF of $\left|h_{s x}\right|$ becomes a Rayleigh PDF and when $m_{s x}=\infty$, it becomes a Rice PDF. The function ${ }_{1} F_{1}(a ; b ; z)$ is the confluent hypergeometric function defined in [12] by

$$
{ }_{1} F_{1}(a ; b ; z)=\sum_{n=0}^{\infty} \frac{(a)_{n}}{(b)_{n}} \frac{z^{n}}{n !}
$$

where $(x)_{n}=x(x+1) \ldots(x+n-1)$.

- The $i^{\text {th }}$ relay-destination link is modeled as a Rayleigh fading channel. The PDF, $f_{\left|h_{r_{i} d}\right|^{2}}(y)$ of the power channel gain, $\left|h_{r_{i} d}\right|^{2}$, is defined in [13] as the exponential distribution

$$
f_{\left|h_{r_{i} d}\right|^{2}}(y)=\frac{1}{2 b_{r_{i} d}} \exp \left(-\frac{y}{2 b_{r_{i} d}}\right) \text {, for } y \geq 0
$$

where $2 b_{r_{i} d}$ is the average power of the multipath component of the $i^{t h}$ relay-destination link.

\section{Outage Analysis}

In this section, we analyze the outage probability of an HSTCS. First, we evaluate the outage probability of the direct link and then we evaluate the outage probability of the system with best relay selection.

\section{A. Outage probability of the direct link}

The instantaneous received signal-to-noise ratio (SNR) of the direct link at the receiver is given by

$$
\gamma_{s d}=\left|h_{s d}\right|^{2} \times \bar{\gamma}_{s d}
$$

where $\bar{\gamma}_{s d}=E_{s} / N_{0}$, is the average transmit SNR per symbol of the satellite-destination link. So, the PDF of $\gamma_{s d}$ can be written as

$$
\begin{aligned}
f_{\gamma_{s d}}(y) & =\frac{1}{\bar{\gamma}_{s d}} \times f_{\left|h_{s d}\right|^{2}}\left(\frac{y}{\bar{\gamma}_{s d}}\right) \\
& =\frac{1}{2 b_{s d} \bar{\gamma}_{s d}}\left(\frac{2 b_{s d} m_{s d}}{2 b_{s d} m_{s d}+\Omega_{s d}}\right)^{m_{s d}} \exp \left(-\frac{y}{2 b_{s d} \bar{\gamma}_{s d}}\right) \\
& \times{ }_{1} F_{1}\left(m_{s d} ; 1 ; \frac{\Omega_{s d} y}{2 b_{s d} \bar{\gamma}_{s d}\left(2 b_{s d} m_{s d}+\Omega_{s d}\right)}\right), \text { for } y \geq 0 .
\end{aligned}
$$


The instantaneous mutual information $I_{s d}$ of the direct link is given by

$$
I_{s d}=\frac{1}{2} \log _{2}\left(1+\gamma_{s d}\right)=\frac{1}{2} \log _{2}\left(1+\frac{E_{s}}{N_{0}}\left|h_{s d}\right|^{2}\right) .
$$

The outage probability $P_{s d}^{o u t}$ of the direct link is then defined as

$$
\begin{aligned}
P_{s d}^{\text {out }} & =\operatorname{Pr}\left[I_{s d}<R\right]=\operatorname{Pr}\left[\gamma_{s d}<2^{2 R}-1\right] \\
& =\operatorname{Pr}\left[\gamma_{s d}<\gamma_{t h}\right]=F_{\gamma_{s d}}\left(\gamma_{t h}\right)
\end{aligned}
$$

where $R$ denotes the target spectral efficiency (in bits/s/Hz), $\gamma_{t h}=2^{2 R}-1$ and $F_{\gamma_{s d}}(y)$ denotes the cumulative distribution function (CDF) of $\gamma_{s d}$. We have that

$$
F_{\gamma_{s d}}(y)=\int_{0}^{y} f_{\gamma_{s d}}(\tau) d \tau
$$

By using the Maclaurin series expansions of $\exp (-x)$ and the table of integrals in [12], the CDF of $\gamma_{s d}$ is obtained as in (8). where

$$
\begin{aligned}
A_{s d} & =\frac{1}{2 b_{s d} \bar{\gamma}_{s d}}\left(\frac{2 b_{s d} m_{s d}}{2 b_{s d} m_{s d}+\Omega_{s d}}\right)^{m_{s d}} \\
B_{s d} & =\frac{\Omega_{s d}}{2 b_{s d} \bar{\gamma}_{s d}\left(2 b_{s d} m_{s d}+\Omega_{s d}\right)}
\end{aligned}
$$

and

$$
{ }_{p} F_{q}\left(a_{1}, \ldots, a_{p} ; b_{1}, \ldots, b_{q} ; z\right)=\sum_{n=0}^{\infty} \frac{\left(a_{1}\right)_{n} \ldots\left(a_{p}\right)_{n}}{\left(b_{1}\right)_{n} \ldots\left(b_{q}\right)_{n} n !} z^{n}
$$

is the general hypergeometric function defined in [12]. Hence, the outage probability of the direct link is given as in (9).

\section{B. Outage probability of the HSTCS with best relay selection}

The instantaneous received SNR at the output of the MRC is given as

$$
\gamma_{M R C}=\gamma_{s d}+\max _{i \in C}\left(\gamma_{r_{i} d}\right)
$$

where $C$ is the decoding set with cardinality $|C| \leq L$. Actually, it will be difficult to find the PDF of $\gamma_{M R C}$ given in (10) because the decoding set $C$ is unknown. To treat this problem we invoke the technique described in [14], where the system in Fig. 1 can be considered as a communication system consisting of $L+1$ effective paths between the satellite and the destination. Let path number 0 be the $s$ to $d$ direct link and path $i$ be the $s \rightarrow r_{i} \rightarrow d$ relayed link where $i=1, \ldots, L$. Let $\chi_{i}$ be the instantaneous received SNR of the relayed link $i$ at the destination which takes into account both the $s$ to $r_{i}$ and the $r_{i}$ to $d$ link. Therefore, the PDF of $\chi_{i}$ is given by

$$
\begin{aligned}
f_{\chi_{i}}(y) & =f_{\chi_{i} \mid r_{i}} \text { Decodes Incorrectly }(y) \operatorname{Pr}\left[r_{i} \text { Decodes Incorrectly }\right] \\
& +f_{\chi_{i} \mid r_{i}} \text { Decodes Correctly }(y) \operatorname{Pr}\left[r_{i} \text { Decodes Correctly }\right]
\end{aligned}
$$

So, equation (10) is equivalent to (12)

$$
\gamma_{M R C}=\gamma_{s d}+\max _{i \in L}\left(\chi_{i}\right)=\gamma_{s d}+\tilde{\gamma}_{D F}
$$

where

$$
\tilde{\gamma}_{D F}=\max _{i \in L}\left(\chi_{i}\right) .
$$

The probability that the relay $r_{i}$ decodes incorrectly is the outage probability, $P_{s r_{i}}^{\text {out }}$, of the satellite-relay $r_{i}$ link and the probability that the relay $r_{i}$ decodes correctly is $\left(1-P_{s r_{i}}^{o u t}\right)$.
This $P_{s r_{i}}^{\text {out }}$ can be calculated by using the same approach as in (9) and is given as in (13). where

$$
\begin{aligned}
A_{s r_{i}} & =\frac{1}{2 b_{s r_{i}} \bar{\gamma}_{s r_{i}}}\left(\frac{2 b_{s r_{i}} m_{s r_{i}}}{2 b_{s r_{i}} m_{s r_{i}}+\Omega_{s r_{i}}}\right)^{m_{s r_{i}}} \\
B_{s r_{i}} & =\frac{\Omega_{s r_{i}}}{2 b_{s r_{i}} \bar{\gamma}_{s r_{i}}\left(2 b_{s r_{i}} m_{s r_{i}}+\Omega_{s r_{i}}\right)} .
\end{aligned}
$$

The conditional PDF $f_{\chi_{i} \mid r_{i}}$ Decodes Incorrectly $(y)$ is given by

$$
f_{\chi_{i} \mid r_{i} \text { Decodes Incorrectly }}(y)=\delta(y), y \geq 0
$$

where $\delta(y)$ is the Dirac Delta function. And the conditional PDF $f_{\chi_{i} \mid r_{i}}$ Decodes Correctly $(y)$ is given by, for $y \geq 0$,

$$
f_{\chi_{i} \mid r_{i} \text { Decodes Correctly }}(y)=\frac{1}{2 b_{r_{i} d} \bar{\gamma}_{r_{i} d}} \exp \left(-\frac{y}{2 b_{r_{i} d} \bar{\gamma}_{r_{i} d}}\right),
$$

where $\bar{\gamma}_{r_{i} d}=E_{r_{i}} / N_{0}$ is the average transmit SNR per symbol of the relay $r_{i}$. Therefore, equation (11) can be written as, for $y \geq 0$,

$$
f_{\chi_{i}}(y)=P_{s r_{i}}^{\text {out }} \delta(y)+\frac{1-P_{s r_{i}}^{\text {out }}}{2 b_{r_{i} d} \bar{\gamma}_{r_{i} d}} \exp \left(-\frac{y}{2 b_{r_{i} d} \bar{\gamma}_{r_{i} d}}\right) .
$$

And the CDF of $\chi_{i}$ can be written as

$$
F_{\chi_{i}}(y)=1-\left(1-P_{s r_{i}}^{\text {out }}\right) \exp \left(-\frac{y}{2 b_{r_{i} d} \bar{\gamma}_{r_{i} d}}\right) .
$$

The CDF of $\tilde{\gamma}_{D F}$ can be computed as follow

$$
\begin{aligned}
F_{\tilde{\gamma}_{D F}}(y) & =\operatorname{Pr}\left[\max _{i \in L}\left(\chi_{i}\right) \leq y\right] \\
& =\prod_{i=1}^{L} \operatorname{Pr}\left(\chi_{i} \leq y\right)=\prod_{i=1}^{L} F_{\chi_{i}}(y) .
\end{aligned}
$$

By using the following property,

$\prod_{k=1}^{L}\left(1+A_{k}\right)=1+\sum_{k=1}^{L} \sum_{\lambda_{1}=1}^{L-k+1} \sum_{\lambda_{2}=\lambda_{1}+1}^{L-k+2} \ldots \sum_{\lambda_{k}=\lambda_{k-1}+1}^{L} \prod_{n=1}^{k} A_{\lambda_{n}}$

the CDF of $\tilde{\gamma}_{D F}$ can be rewritten as in (20).

It is well known that the PDF of the sum of two independent random variables is the convolution product of these two variables. Therefore, the PDF of $\gamma_{M R C}$ is given as

$$
f_{\gamma_{M R C}}(y)=\int_{-\infty}^{+\infty} f_{\tilde{\gamma}_{D F}}(y-\tau) f_{\gamma_{s d}}(\tau) d \tau
$$

After some mathematical manipulations, the CDF of $\gamma_{M R C}$ can be expressed as

$$
F_{\gamma_{M R C}}(y)=\int_{0}^{y} F_{\tilde{\gamma}_{D F}}(y-\tau) f_{\gamma_{s d}}(\tau) d \tau .
$$

By using the same approach as in (8), the CDF of $\gamma_{M R C}$, $F_{\gamma_{M R C}}(y)$, can be obtained as equation (23). So, the outage probability of the HSTCS with best relay selection is finally given as in (24).

In the case of independent and identically distributed fading channels, all relays are experiencing the same fading environment, i.e., $m_{s r_{i}}=m_{s r}, b_{s r_{i}}=b_{s r}, \Omega_{s r_{i}}=\Omega_{s r}, b_{r_{i} d}=b_{r d}$ 


$$
F_{\gamma_{s d}}(y)=A_{s d} y_{1} F_{1}\left(m_{s d} ; 2 ; B_{s d} y\right)+\sum_{j=1}^{+\infty}(-1)^{j} \frac{A_{s d} y^{(j+1)}}{(j+1) !\left(2 b_{s d} \bar{\gamma}_{s d}\right)^{j}}{ }^{2} F_{2}\left(j+1, m_{s d} ; j+2,1 ; B_{s d} y\right)
$$

$$
P_{s d}^{o u t}=A_{s d} \gamma_{t h 1} F_{1}\left(m_{s d} ; 2 ; B_{s d} \gamma_{t h}\right)+\sum_{j=1}^{+\infty}(-1)^{j} \frac{A_{s d}\left(\gamma_{t h}\right)^{(j+1)}}{(j+1) !\left(2 b_{s d} \bar{\gamma}_{s d}\right)^{j}}{ }^{2} F_{2}\left(j+1, m_{s d} ; j+2,1 ; B_{s d} \gamma_{t h}\right)
$$

$$
P_{s r_{i}}^{\text {out }}=A_{s r_{i}} \gamma_{t h 1} F_{1}\left(m_{s r_{i}} ; 2 ; B_{s r_{i}} \gamma_{t h}\right)+\sum_{j=1}^{+\infty}(-1)^{j} \frac{A_{s r_{i}}\left(\gamma_{t h}\right)^{(j+1)}}{(j+1) !\left(2 b_{s r_{i}} \bar{\gamma}_{s r_{i}}\right)^{j}}{ }^{2} F_{2}\left(j+1, m_{s r_{i}} ; j+2,1 ; B_{s r_{i}} \gamma_{t h}\right)
$$

$$
F_{\tilde{\gamma}_{D F}}(y)=1+\sum_{k=1}^{L}(-1)^{k} \sum_{\lambda_{1}=1}^{L-k+1} \sum_{\lambda_{2}=\lambda_{1}+1}^{L-k+2} \ldots \sum_{\lambda_{k}=\lambda_{k-1}+1}^{L} \prod_{n=1}^{k}\left(1-P_{s r_{\lambda_{n}}}^{\text {out }}\right) \exp \left(-\frac{y}{2 b_{r_{\lambda_{n}} d} \bar{\gamma}_{r_{\lambda_{n}} d}}\right)
$$

$$
\begin{aligned}
F_{\gamma_{M R C}}(y) & =F_{\gamma_{s d}}(y)+A_{s d} \sum_{k=1}^{L}(-1)^{k} \sum_{\lambda_{1}=1}^{L-k+1} \sum_{\lambda_{2}=\lambda_{1}+1}^{L-k+2} \ldots \sum_{\lambda_{k}=\lambda_{k-1}+1}^{L}\left[\prod_{n=1}^{k}\left(1-P_{s r_{\lambda_{n}}}^{\text {out }}\right)\right] \exp \left(-\sum_{n=1}^{k} \frac{y}{2 b_{r_{\lambda_{n}} d} \bar{\gamma}_{r_{\lambda_{n}} d}}\right) \\
& \times\left[y_{1} F_{1}\left(m_{s d} ; 2 ; B_{s d} y\right)+\sum_{j=1}^{+\infty}(-1)^{j}\left(\frac{1}{2 b_{s d} \bar{\gamma}_{s d}}-\sum_{n=1}^{k} \frac{1}{2 b_{r_{\lambda_{n}} d \bar{\gamma}_{r_{\lambda_{n}} d}}}\right)^{j} \frac{y^{(j+1)}(j+1) !}{\left(j{ }^{2}\right.} F_{2}\left(j+1, m_{s d} ; j+2,1 ; B_{s d} y\right)\right]
\end{aligned}
$$

$$
\begin{aligned}
P_{\gamma_{M R C}}^{\text {out }} & =P_{s d}^{\text {out }}+A_{s d} \sum_{k=1}^{L}(-1)^{k} \sum_{\lambda_{1}=1}^{L-k+1} \sum_{\lambda_{2}=\lambda_{1}+1}^{L-k+2} \ldots \sum_{\lambda_{k}=\lambda_{k-1}+1}^{L}\left[\prod_{n=1}^{k}\left(1-P_{s \lambda_{\lambda_{n}}}^{\text {out }}\right)\right] \exp \left(-\sum_{n=1}^{k} \frac{\gamma_{t h}}{2 b_{r_{\lambda_{n}} d} \bar{\gamma}_{r_{\lambda_{n}} d}}\right) \\
& \times\left[\gamma_{t h 1} F_{1}\left(m_{s d} ; 2 ; B_{s d} \gamma_{t h}\right)+\sum_{j=1}^{+\infty}(-1)^{j}\left(\frac{1}{2 b_{s d} \bar{\gamma}_{s d}}-\sum_{n=1}^{k} \frac{1}{2 b_{r_{\lambda_{n}} d \bar{\gamma}_{\lambda_{\lambda_{n}} d}}}\right)^{j} \frac{\gamma_{t h}^{(j+1)}(j+1) !}{\left(j+F_{2}\right.} F_{2}\left(j+1, m_{s d} ; j+2,1 ; B_{s d} \gamma_{t h}\right)\right]
\end{aligned}
$$

$$
\begin{aligned}
P_{\gamma_{M R C}}^{\text {out }} & =P_{s d}^{\text {out }}+A_{s d} \sum_{k=1}^{L}\left(\begin{array}{l}
L \\
k
\end{array}\right)(-1)^{k}\left(1-P_{s r}^{\text {out }}\right)^{k} \exp \left(-\frac{k}{2 b_{r d} \bar{\gamma}_{r d}} \gamma_{t h}\right) \\
& \times\left[\gamma_{t h 1} F_{1}\left(m_{s d} ; 2 ; B_{s d} \gamma_{t h}\right)+\sum_{j=1}^{+\infty}(-1)^{j}\left(\frac{1}{2 b_{s d} \bar{\gamma}_{s d}}-\frac{k}{2 b_{r d} \bar{\gamma}_{r d}}\right)^{j} \frac{\gamma_{t h}^{(j+1)}}{(j+1) !}{ }^{2} F_{2}\left(j+1, m_{s d} ; j+2,1 ; B_{s d} \gamma_{t h}\right)\right]
\end{aligned}
$$

and $\bar{\gamma}_{r_{i} d}=\bar{\gamma}_{r d}$ for all $i \in L$. Then the outage probability, $P_{\gamma_{M R C}}^{\text {out }}$, is reduced to (25).

Although the outage probability in (24) and (25) is expressed as an infinite sum of general hypergeometric functions, it is convergent when the number of terms in the sum of the second line of (24) and (25) is high enough, i.e., $j \gg 1$.

\section{Simulation Results}

In this section, we present the numerical results of the outage probability of an HSTCS with best relay selection versus the average transmit $\mathrm{SNR}, E_{s} / N_{0}$, for different number of participating relays $L=0,1,2,3$ ( $L=0$, corresponds to the direct transmission only). The numerical values for the LMS channel are shown in Table I and the relay-destination links are Rayleigh fading channels. The target spectral efficiency $R$ is assumed to be $0.5 \mathrm{bits} / \mathrm{s} / \mathrm{Hz}$. In order to show the diversity order, we assume that the average transmit SNR per symbol of the satellite-destination link is equal to the one of the relaysdestination link $\left(E_{s} / N_{0}=E_{r_{i}} / N_{0}\right)$. The figures illustrate that our analytical results (for $j=20$ ) show excellent agreement with the simulation results for both independent non identically distributed fading channels (Fig. 2) and independent identically distributed fading channels (Fig. 3).

Fig. 2 shows the outage probability of an HSTCS with best relay selection when the direct link experiences the frequent heavy shadowing. The first, the second and the third satelliterelay links experience the average shadowing, the infrequent 
TABLE I

LMS CHANNEL PARAMETERS [11]

\begin{tabular}{c|c|c|c|}
\multirow{2}{*}{} & \multicolumn{3}{|c|}{ Data set } \\
\cline { 2 - 4 } & $\mathrm{b}$ & $\mathrm{m}$ & $\Omega$ \\
\hline Frequent heavy shadowing & 0.063 & 0.739 & $8.97 \times 10^{-4}$ \\
\hline Average shadowing & 0.126 & 10.1 & 0.835 \\
\hline Infrequent light shadowing & 0.158 & 19.4 & 1.29 \\
\hline
\end{tabular}

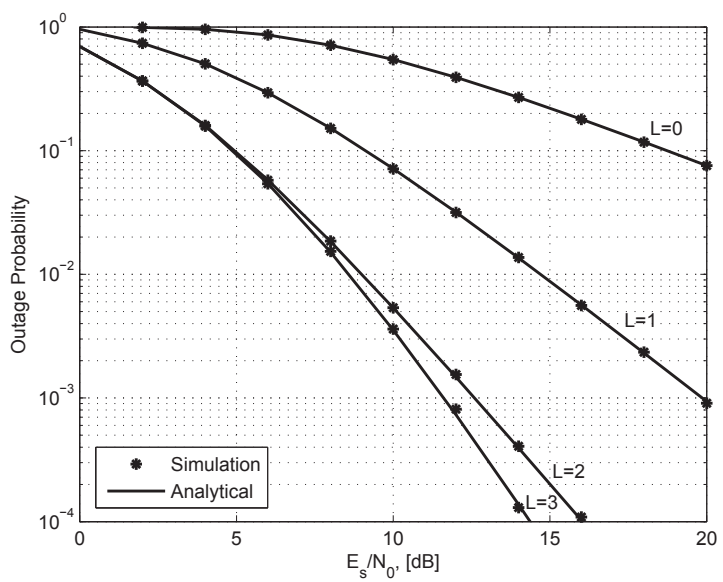

Fig. 2. The outage probability of an HSTCS with best relay selection versus the average transmit SNR, $E_{s} / N_{0}$, when the direct link experiences the frequent heavy shadowing. The first, the second and the third satellite-relays experience the average shadowing, the infrequent light shadowing and the frequent heavy shadowing respectively while their terrestrial links experience the Rayleigh fading with the average power channel gain equal to $0.5,1$ and 0.25 respectively.

light shadowing and the frequent heavy shadowing while its terrestrial links experience the Rayleigh fading with the average power channel gain equal to $0.5,1$ and 0.25 respectively. We observe from the figure that the 3-relay system curve and the 2-relay system curve are superimposed in the lowSNR regime. This is because of the strong fading of the third relay link. We can observe in the figure that the one-relay system provides a diversity gain of $10 \mathrm{~dB}$ at the outage rate of $10^{-1}$ over the direct link while the 2-relay system provides a diversity gain of $14 \mathrm{~dB}$ over the direct link at the same outage rate.

Fig. 3 shows the outage probability of an HSTCS with best relay selection when both direct link and satellite-relay links experience the frequent heavy shadowed fading while all terrestrial links are Rayleigh with average channel power gain equal to unity. We can achieve approximately $5.5 \mathrm{~dB}$ of diversity gain at the outage probability of $10^{-1}$ when only one relay is participating. In addition, this diversity gain does increase to approximately $9 \mathrm{~dB}$ when $L=3$.

\section{CONCLUSION}

In this paper, the closed-form expressions for the outage probability of an HSTCS with best relay selection over independent but not necessarily identically distributed fading channels have been derived. The results have shown that a full diversity order of $L+1$ can be obtained when the

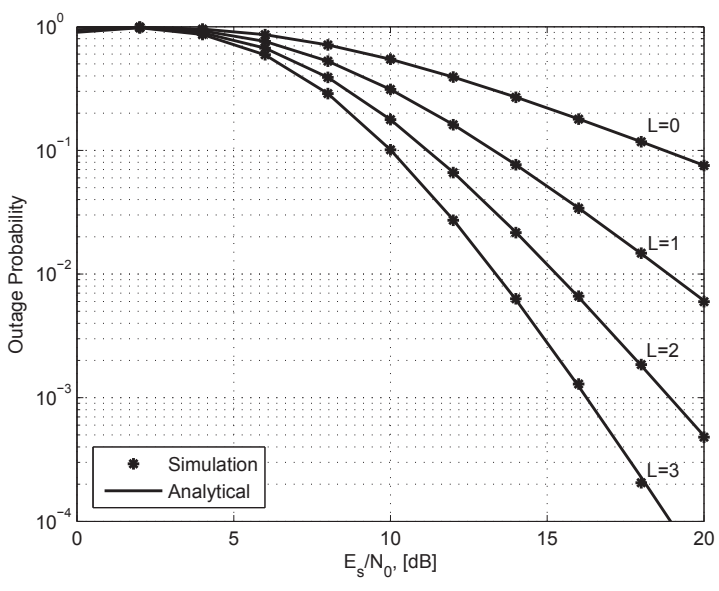

Fig. 3. The outage probability of an HSTCS with best relay selection versus the average transmit $\mathrm{SNR}, E_{S} / N_{0}$, when the direct link and the relay links experience the frequent heavy shadowing.

number of participating relays is equal to $L$. Moreover, it can be seen from the outage curves that simulation results confirm analytical results. The obtained outage expressions will provide valuable insight into the design of an HSTCS.

\section{REFERENCES}

[1] G. G. Kota, S. and S. Kim, "Satellite component of NGN: Integrated and hybrid networks," Int. J. Sat. Commun., vol. 29, pp. 191-208, 2010.

[2] G. Iapichino, C. Bonnet, O. del Rio Herrero, C. Baudoin, and I. Buret, "Advanced hybrid satellite and terrestrial system architecture for emergency mobile communications," in ICSSC'08 AIAA, Jun. 2008, pp. 1-8.

[3] S. Morosi, S. Jayousi, and E. Del Re, "Cooperative delay diversity in hybrid satellite/terrestrial DVB-SH system," in IEEE ICC'10, May. 2010, pp. $1-5$.

[4] D.-S. Ahn, S. Kim, H. W. Kim, and D.-C. Park, "A cooperative transmit diversity scheme for mobile satellite broadcasting systems." Int. J. Sat. Commun., vol. 28, pp. 352-368, 2010.

[5] B. Paillassa, B. Escrig, R. Dhaou, M.-L. Boucheret, and C. Bes, "Improving satellite services with cooperative communications," Int. J. Sat. Commun., vol. 29, pp. 479-500, 2011.

[6] A. Bletsas, A. Khisti, D. P. Reed, and A. Lippman, "A simple cooperative diversity method based on network path selection," IEEE J. Sel. Areas Commun., vol. 24, no. 3, pp. 659 -672, 2006.

[7] X. Chen, T. wai Siu, Q. Zhou, and F. Lau, "High-SNR analysis of opportunistic relaying based on the maximum harmonic mean selection criterion," IEEE Signal Process. Lett., vol. 17, no. 8, pp. $719-722$, Aug. 2010.

[8] B. Escrig, "On-demand cooperation MAC protocols with optimal diversity-multiplexing tradeoff," in IEEE Wireless Commun. and Netw. Conf., Apr. 2010, pp. 1-6.

[9] Y.-C. Ko, M.-S. Alouini, and M. Simon, "Outage probability of diversity systems over generalized fading channels," IEEE Trans. Commun., vol. 48 , no. 11 , pp. 1783 - 1787, Nov. 2000.

[10] A. Iqbal and K. M. Ahmed, "A hybrid satellite-terrestrial cooperative network over non identically distributed fading channels," Journal of communications, vol. 7, pp. 581-589, 2011.

[11] A. Abdi, W. Lau, M.-S. Alouini, and M. Kaveh, "A new simple model for land mobile satellite channels: first- and second-order statistics," IEEE Trans. Wireless Commun., vol. 2, no. 3, pp. 519 - 528, May 2003.

[12] I. S. Gradshteyn and I. M. Ryzhik, Table of Integrals, Series, and Products. New York: Academic Press, 2007.

[13] M. K. Simon and M.-S. Alouini, Digital Communication over Fading Channels. Wiley, 2005.

[14] N. C. Beaulieu and J. Hu, "A closed-form expression for the outage probability of decode-and-forward relaying in dissimilar Rayleigh fading channels," IEEE Commun. Lett., vol. 10, no. 12, pp. $813-815$, Dec. 2006. 